\title{
THE CONVERGENCE OF LEAST SQUARES APPROXIMATIONS FOR DUAL ORTHOGONAL SERIES
}

\author{
by ROBERT P. FEINERMAN and ROBERT B. KELMAN
}

(Received 9 January, 1973)

1. Introduction. The convergence of least squares approximations for dual orthogonal series in Hilbert space is established, thus providing a theorem applicable to practically all dual orthogonal series (such as dual trigonometric series, dual Bessel series, etc.) that have appeared in the literature. Our results establish for such dual series the existence of a sequence of functions satisfying in the $L^{2}$ norm the dual series relation, with an error tending to zero and, in particular, rigorously justify the calculations in [2] which showed least squares to be a practical approximation procedure for dual trigonometric equations. In fact, the desire to provide a rigorous convergence theorem for [2] motivated this study.

Standard definitions and notation for Hilbert space are employed [1]. We denote by $\mathbf{R}$ a real, separable, abstract Hilbert space. The subspaces $\mathbf{P}$ and $\mathbf{Q}$ are orthogonal complements, while $P$ and $Q$ denote respectively the projection operators from $\mathbf{R}$ onto $\mathbf{P}$ and onto $\mathbf{Q}$, We recall that $P+Q$ is the identity operator. $\left\{\phi_{n}: n=1,2, \ldots\right\}$ denotes a complete orthonormal sequence in $\mathbf{R}$, while $\psi=\left\{\psi_{n}\right\}$ is defined by $\psi_{n}=a_{n} P \phi_{n}+b_{n} Q \phi_{n}$, where $\left\{a_{n}\right\}$ and $\left\{b_{n}\right\}$ are real, nonnegative sequences. In $\mathbf{R}$ the dual orthogonal series problem is this: Given $\left\{a_{n}\right\}$, $\left\{b_{n}\right\},\left\{\phi_{n}\right\}$ and $f \in \mathbf{R}$, find a real sequence $\left\{j_{n}\right\}$ such that $f=\sum_{1}^{\infty} j_{n} \psi_{n}$. The dual orthogonal series approximation problem is this: With the above assumptions, find a sequence of vectors $\left(j_{1}^{N}, j_{2}^{N}, \ldots, j_{N}^{N}\right) \quad(N=1,2, \ldots)$ such that

$$
\lim _{N \rightarrow \infty} F_{N} \equiv \lim _{N \rightarrow \infty}\left\|\sum_{n=1}^{N} j_{n}^{N} \psi_{n}-f\right\|^{2}=0 .
$$

In this paper we study the approximation problem.

2. Convergence theorem. We proceed to

THEOREM 1. Let $\left\{b_{n}\right\}$ be a sequence of positive numbers. Let $\left\{a_{n}\right\}$ be such that either (i)

$$
a_{n}>0 \quad(n=1,2, \ldots),
$$

or (ii) there is a positive integer $K$ such that

$$
\begin{gathered}
a_{n}=0 \quad(n=1,2, \ldots, K) \quad \text { and } \quad a_{n}>0 \quad(n=K+1, K+2, \ldots), \\
D \equiv\left|\left(\phi_{m}, D \phi_{n}\right): m, n=1,2, \ldots, K\right| \neq 0,
\end{gathered}
$$

where $D$ is a $K \times K$ determinant.

Then there is a unique sequence of vectors $\left(j_{1}^{N}, j_{2}^{N}, \ldots, j_{N}^{N}\right)(N=1,2, \ldots)$, which minimize the quadratic form $F_{N}$ and for which (1) is valid. These vectors are the unique solutions of the systems of linear algebraic equations 


$$
\sum_{n=1}^{N}\left(\psi_{n}, \psi_{m}\right) j_{n}=\left(f, \psi_{m}\right) \quad(m=1,2, \ldots, N)
$$

REMARK. The assumptions (3) and (4) are needed for certain dual orthogonal series associated with problems involving a zero eigenvalue, e.g., potential problems with periodic boundary conditions or Neumann conditions (cf. equations 11A, 11B).

Proof. We prove the theorem assuming (3) and (4), the proof using (2) being a special case. First we show that $\psi$ is complete, i.e. that, if

$$
\left(f, \psi_{n}\right)=0 \quad(n=1,2, \ldots)
$$

then $f=0$. Let $\sum_{1}$ denote summation over all positive integers $n$ such that $b_{n}<2 a_{n}$, and $\sum_{2}$ summation over all other positive integers. Assume that $f$ satisfies (6). Using Parseval's identity and a little manipulation, we obtain

$$
\|f\|^{2}=\sum_{1}\left(1-\left(b_{n} / a_{n}\right)\right)^{2}\left(f, Q \phi_{n}\right)^{2}+\sum_{2}\left(1-\left(a_{n} / b_{n}\right)\right)^{2}\left(f, P \phi_{n}\right)^{2} .
$$

If, for some $n>K$, one has $\left(f, \phi_{n}\right) \neq 0$, then

$$
\|f\|^{2}<\sum_{1}\left(f, Q \phi_{n}\right)^{2}+\sum_{2}\left(f, P \phi_{n}\right)^{2} \leqq\|Q f\|^{2}+\|P f\|^{2}=\|f\|^{2} .
$$

Thus, without loss of generality, we can assume that $f=\sum_{n=1}^{K} j_{n} \phi_{n}$. This combined with (6) shows that

$$
\sum_{n=1}^{K}\left(\phi_{m}, Q \phi_{n}\right) j_{n}=0 \quad(m=1,2, \ldots, K) .
$$

Condition (4) implies that $j_{n}=0(n=1,2, \ldots, K)$. Therefore $\psi$ is complete.

Next we show that $\psi$ is finitely linearly independent, i.e. that the elements $\psi_{1}, \psi_{2}, \ldots, \psi_{n}$ are linearly independent, for any $n$. Let us assume that there is a vector $k=\left(k_{1}, k_{2}, \ldots, k_{L}\right)$ such that $\sum_{n=1}^{L} k_{n} \psi_{n}=0$. We can assume that $L \geqq K$ without loss of generality. Separating the orthogonal components yields

$$
\sum_{n=1}^{L} k_{n} a_{n} P \phi_{n}=0 \quad \text { and } \quad \sum_{n=1}^{L} k_{n} b_{n} Q \phi_{n}=0 .
$$

Let us define the $L \times L$ matrices $S$ and $T$, where $S$ is diagonal, by

$$
S_{n n}=a_{n} b_{n} \quad \text { and } T_{m n}=\left(Q \phi_{m}, \phi_{n}\right)\left(a_{m} b_{n}-a_{n} b_{m}\right) \quad(m, n=1,2, \ldots, L) \text {. }
$$

We multiply both sides of the first equation in (9) by $b_{m}$ and both sides of the second equation by $a_{m}$ and then form inner products with $\phi_{m}$. Upon adding both sides of the resulting equations we obtain $T k=-S k$. One finds that $(T k, k)=0$ for the inner product in euclidean $L$-space, since $T_{m n}=-T_{n m}$. Thus $(S k, k)=0$, so that $k_{n}=0$ if $n>K$. Returning to (9) and setting $j_{n}=b_{n} k_{n}$ we again obtain (8). Thus $k=0$, and $\psi$ is linearly independent.

Since $\psi$ is çomplete and linearly independent, it follows forthwith [3, p. 225] that equations (5) have a unique solution which minimizes $F_{N}$ and for which (1) is valid. This ends the proof. 
3. Illustrative example. To illustrate the application of Theorem 1 to a specific problem we consider the dual Legendre series

$$
\begin{aligned}
& \sum_{n=2}^{\infty} j_{n}(n-1)\left(\frac{2 n-1}{2}\right)^{1 / 2} P_{n-1}(x)=g(x) \quad(-1 \leqq x<c), \\
& \sum_{n=1}^{\infty} j_{n}\left(\frac{2 n-1}{2}\right)^{1 / 2} P_{n-1}(x)=g(x) \quad(c<x \leqq 1),
\end{aligned}
$$

where $P_{n}$ is a Legendre polynomial and $g \in L^{2}(-1,1)$. (This dual series arises upon seeking the steady temperature in a sphere with part of the spherical surface subjected to a Neumann condition and part to a Dirichlet condition.) Here $L^{2}(-1,1)$ is the realization of $\mathbf{R}$, while $\mathbf{P}$ consists of all functions $h \in L^{2}(-1,1)$ such that $h=0$, almost everywhere, on $c<x<1$. It follows that $\mathbf{Q}$ consists of all $h \in L^{2}(-1,1)$ such that $h=0$ almost everywhere on $-1<x<c$, and that $P h=h$ for $-1 \leqq x<c$ and $P h=0$ for $c<x \leqq 1$. Here $g(x)$ is the realization of $f$, and

$$
\phi_{n}=\left(\frac{2 n-1}{2}\right)^{1 / 2} P_{n-1}(x) .
$$

Finally, we have $a_{n}=n-1$ and $b_{n}=1$. We find that $D=(1-c) / 2$ for $K=1$, so that (4) is satisfied. Since $\left\{\phi_{n}\right\}$ as given by (12) is a complete orthonormal sequence in $L^{2}(-1,1)$, the dual Legendre equation (11A, B) satisfies the hypothesis of Theorem 1.

4. Conclusion. Using the example above as a guide, one easily verifies that most dual series equations in the literature satisfy the hypothesis of Theorem 1 , as we have found in reviewing some sixty papers on the subject. The least squares procedure is numerically implemented in [2], where equation (3) corresponds to equation (5) here-the key equation for computing. Theorem 1 implies nothing about the limit properties of the sequence $\left(j_{1}^{N}, j_{2}^{N}, \ldots, j_{N}^{N}\right)$. The relation of this to the approximation problem for variational procedures is discussed in $[3$, Chapter $3, \S 7]$ and the references therein. In a manuscript in preparation entitled " Dual orthogonal series", we show that, if $a_{n} / b_{n}$ tends to a positive limit as $n \rightarrow \infty$, the sequence of least squares approximations converges to a vector $\left(j_{1}, j_{2}, \ldots\right)$ with square summable components. The proof is more elaborate than the straightforward analysis given here and does not apply to the examples here, in [2], and, in general, to dual orthogonal series associated with mixed boundary value problems in which one of the mixed conditions is a Dirichlet condition.

\section{REFERENCES} 1954).

1. N. I. Achiezer and I. M. Glasmann, Theorie der linearen Operatoren in Hilbert-Raum (Berlin,

2. R. B. Kelman and C. A. Koper, Jr, Least squares approximations for dual trigonometric series, Glasgow Math. J. 14 (1973), 111-119.

3. S. G. Mikhlin and K. L. Smolitskiy, Approximate methods for solution of differential and integral equations (New York, 1967).

HeRbert H. Lehman College

BRONX, NEW YORK 10468
Computer Science Section

Colorado State University

Ft. Collins, Colorado 80521 